\title{
Cytoprotective potential of anti-ischemic drugs against chemotherapy-induced cardiotoxicity in H9c2 myoblast cell line
}

\author{
TIAM FERIDOONI ${ }^{1}$ \\ CHRIS MAC DONALD 2 \\ DI $\mathrm{SHAO}^{1}$ \\ POLLEN YEUNG ${ }^{3}$ \\ REMIGIUS U. AGU ${ }^{1 *}$ \\ ${ }^{1}$ Department of Pharmacology \\ Dalhousie University, Halifax, Nova Scotia \\ Canada B3H 4R2, Canada \\ 2 Biopharmaceutics and Drug Delivery \\ Laboratory, Dalhousie University \\ Halifax, NS B3H 3J5, Canada \\ 3 Pharmacokinetics and Metabolism \\ Laboratory, College of Pharmacy, Dalhousie \\ University, Halifax NS B3H 3J5, Canada
}

\begin{abstract}
To investigate potential prevention or attenuation of anti-cancer drug induced cardiotoxicity using anti-ischemic drugs, a rat myoblast $(\mathrm{H} 9 \mathrm{c} 2)$ cell line was used as our in vitro cardiac model. Irinotecan and doxorubicin were found to be cytotoxic for the H9c2 cell line with $I C_{50}$ of $30.69 \pm 6.20$ and $20.94 \pm 6.05 \mu \mathrm{mol} \mathrm{L}^{-1}$, respectively. 5-Flurouracil and cladribine were not cytotoxic and thus $I C_{50}$ could not be calculated. When $100 \mu \mathrm{mol} \mathrm{L}^{-1}$ doxorubicin was incubated for 72 hours with $50 \mu \mathrm{mol} \mathrm{L}^{-1}$ diltiazem, $100 \mu \mathrm{mol} \mathrm{L}^{-1}$ dexrazoxane and $100 \mu \mathrm{mol} \mathrm{L}-1$ losartan, respectively, there was a $58.7 \pm 10.2,52.2 \pm 11.7$ and $44.7 \pm 5.4 \%$ reduction in cell death. When $200 \mu \mathrm{mol}$ $\mathrm{L}^{-1}$ irinotecan was incubated for 72 hours with $100 \mu \mathrm{mol}$ $\mathrm{L}^{-1}$ dexrazoxane, losartan and diltiazem, respectively, a $27.7 \pm 6.9,25.6 \pm 5.1$, and $19.1 \pm 2.3 \%$ reduction in cell death was observed. Our data suggests that losartan and diltiazem were as effective as dexrazoxane in protecting the cells against irinotecan- and doxorubicin-induced cell toxicity. These findings offer potential uses of anti-ischemic drugs for ablation of cytotoxicity in response to mitochondrial injury, thereby improving patient outcomes and reducing health-care costs.
\end{abstract}

Keywords: rat myoblast (H9c2), cytoprotection, dexrazoxane, doxorubicin, diltiazem, irinotecan

Even though new concepts have been introduced with regard to treatment of a variety of cancers, such as various targeted pro-drug and gene therapy approaches, systemic anti-proliferative agents that prevent cellular DNA function are still among the most widely used forms of anti-cancer chemotherapy. Amongst these is the general class of anti-cancer drugs named topoisomerase (Topo) inhibitors (1). Topo I-targeted drugs (e.g., irinotecan) and Topo II-targeted drugs (e.g., doxorubicin), act by stabilizing the covalent enzyme-DNA complex, thereby preventing relegation in the process (2). Increased use of

* Correspondence; e-mail: Remigius.agu@dal.ca 
T. Feridooni et al:: Cytoprotective potential of anti-ischemic drugs against chemotherapy-induced cardiotoxicity in H9c2 myoblast cell line, Acta Pharm. 63 (2013) 493-503.

irinotecan and doxorubicin poses an increased risk of cytotoxicity, resulting from cardiomyocyte apoptosis, myocardial fibrosis, leading to cardiomyopathy, arrhythmias and congestive heart failure (3).

Anthracyclines are highly potent antineoplastic agents for numerous hemopoietic and solid tumors (4). However, their use is limited due to serious cytotoxicity, that has been proven to be fatal. Hence, there is a need for a method that could reduce the cytotoxicity of these agents, thus increasing their use for a larger patient population. The precise mechanism how these chemotherapeutic agents induce cytotoxicity is still unknown, but many theories have been presented, such as the notion that anthracyclines intercalate DNA and promote free radical synthesis which causes tissue damage. Numerous methods have been suggested for effectively blocking doxorubicin-induced cytotoxicity. The suggested methods include inhibition of endocanabiniods, administration of antioxidants and scavengers of peroxynitrite, iron chelating agents and glutamate, use of agents such as erythropoietin or granulocyte stimulating agents, targeted disruption of p53 activity by global gene knockout, chemical inhibition, or cardiomyocyte specific ablation of p53 (5). Another possible method to reduce cytotoxicity is the concept of co-administration of cytoprotectants with the chemotherapeutic agent. This may improve patient outcomes, minimize harm and decrease health care costs. In this study, we investigated the use of cytoprotective drugs in the presence of cardiocytotoxic anti-cancer drugs hoping to reduce cell death.

\section{EXPERIMENTAL}

\section{Chemicals}

Doxorubicin, dexrazoxane, 5-flurouracil, cladribine, and [3-(4,5-dimethylthiazol-2-yl)-2,5-diphenyltetrazolium bromide, MTT] were purchased from Sigma Chemical Co. (USA). Diltiazem, losartan and irinotecan were provided by their developers from Canada Sanofi-Aventis Canada Inc., Merk-Frosst Canada Ltd. and Pfizer Canada Inc., respectively. DMEM/F12 1/1, streptomycin $\left(100 \mu \mathrm{g} \mathrm{mL}^{-1}\right)$, penicillin $\left(100 \mathrm{IU} \mathrm{mL}^{-1}\right)$, physiological saline, trypsin-EDTA and fetal bovine serum (FBS) were from Invitrogen (Canada).

\section{Cell culture}

Rat myoblast (H9c2) cell line was obtained from Cedarlane (Canada). The cells were cultured according to the supplier's protocol and sub-cultured every other day with DMEM/F12 supplemented with $10 \%$ FBS at $37{ }^{\circ} \mathrm{C}$ in a humidified environment $(5 \%$ $\mathrm{CO}_{2} / 95 \% \mathrm{O}_{2}$ ). During passaging, the cells were trypsinized with $0.25 \%$ trypsin-EDTA for not more than $15 \mathrm{~min}$.

\section{Cytotoxicity assay}

Cytoprotection studies were performed on confluent cells in 96-well plates. Chemotherapeutic agents and anti-ischemia drugs were added into the wells and cells were incubated for 72 hours. Concentrated solutions of test compounds were used to avoid up to $10 \%$ dilution of the media supplement used for feeding the cells (10\% FBS in 
T. Feridooni et al.: Cytoprotective potential of anti-ischemic drugs against chemotherapy-induced cardiotoxicity in H9c2 myoblast cell line, Acta Pharm. 63 (2013) 493-503.

DMEM/F12). Cell toxicity was quantified using a MTT assay. Cells grown without cytoprotective drugs served as negative controls while cells grown with dexrazoxane, a clinically approved cytoprotectant, served as a positive control. Cell survival was expressed using simple percentages and $I C_{50}$.

In order to measure the cytotoxicity of anti-cancer drugs, the $\mathrm{H} 9 \mathrm{c} 2$ cells were pre-incubated with various concentrations of doxorubicin or irinotecan for 72 hours. Preliminary studies involving incubation for $12,24,48$ and 72 hours, were performed and showed that incubation for 72 hours gave the most reproducible results and therefore it was used for subsequent studies.

MTT stock solution was made by dissolving $5 \mathrm{mg}$ of thiazolyl blue tetrazolium bromide in $1 \mathrm{~mL}$ of PBS solution. An aliquot of the stock solution $(10 \mu \mathrm{L})$ was added to each well after the test compounds had been incubated at $37^{\circ} \mathrm{C}$ in a humidified environment $\left(5 \% \mathrm{CO}_{2} / 95 \% \mathrm{O}_{2}\right.$ ) for 4 hours to allow the MTT to be converted to colored tetrazolium dye. The medium was then removed and formazan salt crystals were dissolved in 200 $\mu \mathrm{L}$ dimethyl sulfoxide. The absorbance of the wells was measured at $560 \mathrm{~nm}$ using an ELISA plate reader (BioTek Synergy HT, Biotek Instruments, USA).

\section{Fluroescence microscopy}

The rat myoblast $(\mathrm{H} 9 \mathrm{c} 2)$ cell line was suspended in a DMEM/F12 supplemented with $10 \%$ FBS and seeded on a 6-well plate. Stock solutions of test compounds (doxorubicin, irinotecan) were added to the medium, bringing the well volume up to $2 \mathrm{~mL}$ containing $100 \mu \mathrm{mol} \mathrm{L}^{-1}$ of each chemotherapeutic agent. The cells were then incubated at $37{ }^{\circ} \mathrm{C}$ in a humidified environment $\left(5 \% \mathrm{CO}_{2} / 95 \% \mathrm{O}_{2}\right)$ for 72 hours with the anti-ischemic/cytoprotectant or a control involving only the chemotherapeutic agent. At the end of the incubation period, epifluorescence microscopy was performed using a Zeiss Axiovert 10 microscope with oil immersion objectives (Carl Zeiss Ltd., USA). Fluorescence excitation was at $488 \mathrm{~nm}$ and emission at $520 \mathrm{~nm}$.

\section{Data analyses}

Unless stated otherwise, all experiments were performed in 4 replicates and data were presented as mean $\pm S D$. $I C_{50}$ was determined using non-liner regression with Graphpad Prism (San Diego, CA, USA). Change in cell viability was calculated and expressed as percentage. These changes were compared for statistical significance using the analysis of variance (ANOVA) followed by post-test analysis and were considered significant at $p<0.05$.

\section{RESULTS AND DISCUSSION}

\section{Estimation of cardiac cytotoxicity of doxorubicin, irinotecan, 5-flurouracil and cladribine in $\mathrm{H} 9 \mathrm{c} 2$ cells}

The first part of the study involved an estimation of cytotoxicity of selected chemotherapeutic drugs in H9c2 cells. In order to measure the cytotoxicity of anticancer drugs, the H9c2 cells were pre-incubated with various concentrations of doxorubicin or irinotecan for 72 hours. Preliminary studies involving incubation for 12, 24, 48 and 72 
T. Feridooni et al:: Cytoprotective potential of anti-ischemic drugs against chemotherapy-induced cardiotoxicity in H9c2 myoblast cell line, Acta Pharm. 63 (2013) 493-503.

hours, were performed and showed that incubation for 72 hours gave most reproducible results and it was therefore used for subsequent studies (Fig. 1). Consistent with previous studies (6), we observed decreased cell viability with increasing concentration of doxorubicin using MTT and propidium assay. After 72 hours of incubation with doxorubicin at varying concentration, the half maximal inhibitory concentration $\left(I C_{50}\right)$ of doxorubicin was calculated to be $20.94 \pm 6.05 \mu \mathrm{mol} \mathrm{L}^{-1}$ (Fig. 2). Similarly, the $I C_{50}$ of irinotecan was $30.69 \pm 6.19 \mu \mathrm{mol} \mathrm{L}-1$ (Fig. 2). The $I C_{50}$ of 5 -flurouracil and cladribine could not be calculated since the target range tested did not have any toxic effects on the $\mathrm{H} 9 \mathrm{c} 2$ cell line (Fig. 1). Therefore, only doxorubicin and irinotecan were used in subsequent cytoprotection studies.

Once administered, irinotecan is activated as a result of hydrolysis in the liver to SN-38, a Topo-I inhibitor. The SN-38 compound is then inactivated by uridine diphosphate glucoronosyltransferase 1A1 in the hepatic tissue to the corresponding glucuronide (7). However, unlike hepatocytes, most other cells in the body are incapable of detoxifying SN-38 through glucoronidation, resulting in the seen cytotoxicity (8). The inhibitory effect of Topo-II by doxorubicin is well established. Another mechanism for cellular toxicity is that doxorubicin is also able to undergo redox reaction in the mito-

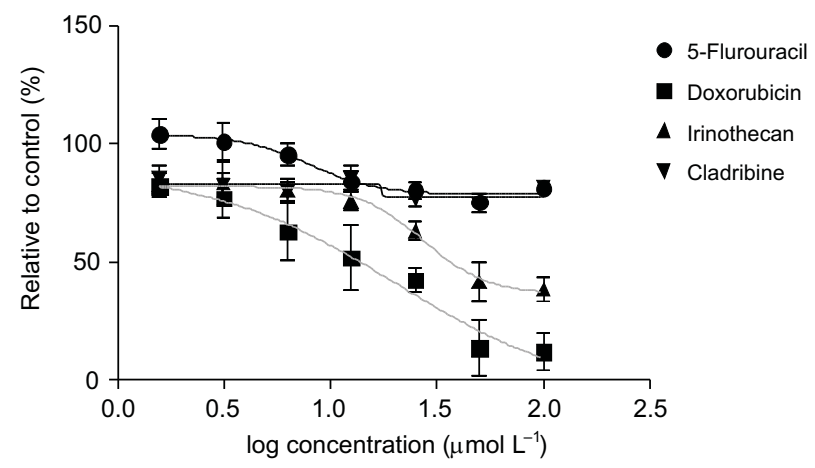

Fig. 1. Dose response curves for doxorubicin, irinotecan, cladribine, and 5-flurouracil. Data are expressed as mean percent of cell survival $\pm \mathrm{SD}(n=4)$.

a)

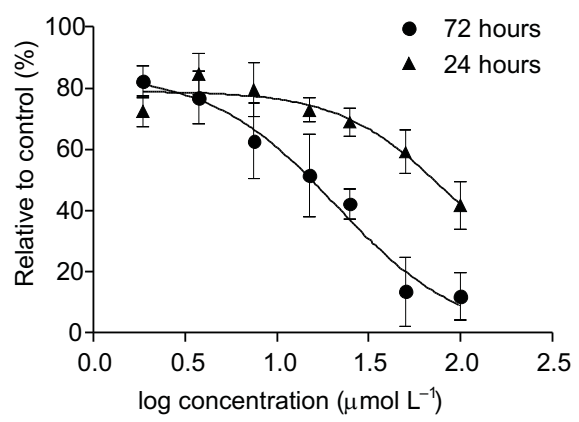

b)

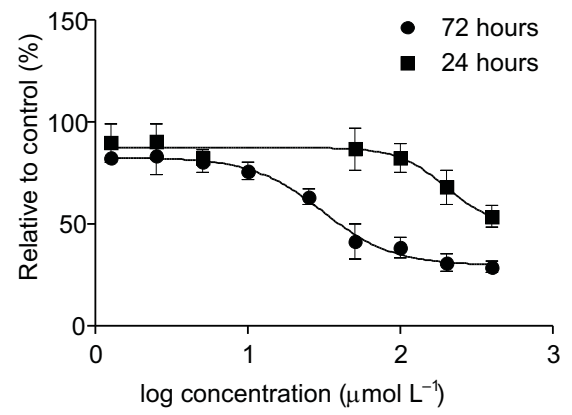

Fig. 2. Effect of incubation time on: a) doxorubicin and b) irinotecan. 
T. Feridooni et al:: Cytoprotective potential of anti-ischemic drugs against chemotherapy-induced cardiotoxicity in H9c2 myoblast cell line, Acta Pharm. 63 (2013) 493-503.

chondria, where it is reduced via NADH dehydrogenase, forming a semiquinone compound (9). The semiquinone compound is then oxidized back to its original structure once its electron is transferred to an oxygen, thus generating a reactive oxygen species (ROS) including hydrogen peroxide and hydroxyl radicals. The generated superoxides then react with nitric oxides in the surrounding tissue generating a reactive nitrogen species (RNS) (5). The build-up of ROS/RNS in cells can cause DNA damage, activation of poly ADP ribose polymerase, up-regulation of redox sensitive transcription factors (p53 and NF- $\mathrm{kB}$ ), and an increase in mitochondrial permeability and apoptosis (10).

\section{Cytoprotective effects of dexrazoxane, losartan and diltiazem against} toxicities induced by doxorubicin and irinotecan in H9c2 cells

After determining the $I C_{50}$ for cytotoxicity of doxorubicin and irinotecan in our assay, the protective effect of the anti-ischemia drugs losartan and diltiazem was assessed. Our first approach was to determine the concentration range that will be used for the

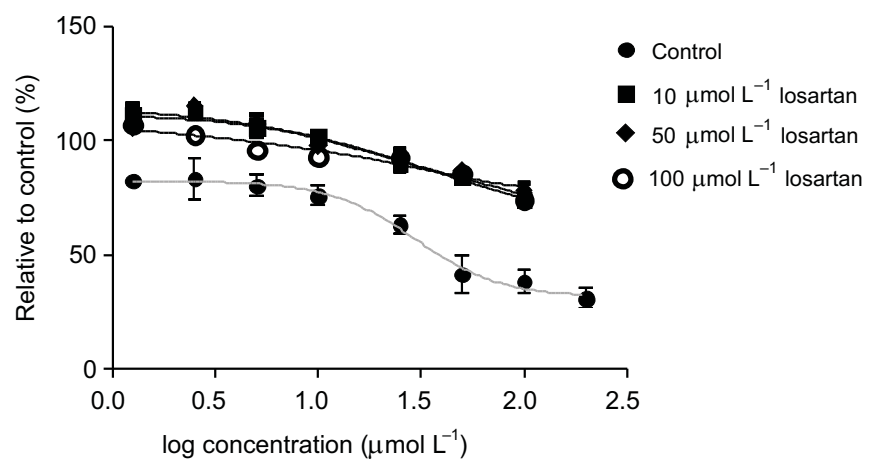

Fig. 3. Effect of dexrazoxane, diltiazem, and losartan cconcentrations on irinotecan-induced cytotoxicity in $\mathrm{H} 9 \mathrm{c} 2$ cells.

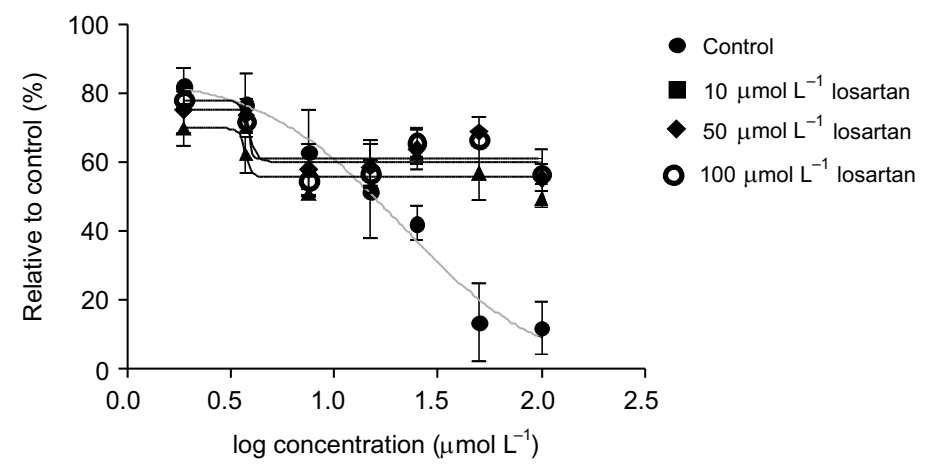

Fig. 4. Effect of dexrazoxane, diltiazem, and losartan concentrations on doxorubicin-induced cytotoxicity in $\mathrm{H} 9 \mathrm{c} 2$ cells. 
T. Feridooni et al: : Cytoprotective potential of anti-ischemic drugs against chemotherapy-induced cardiotoxicity in H9c2 myoblast cell line, Acta Pharm. 63 (2013) 493-503.

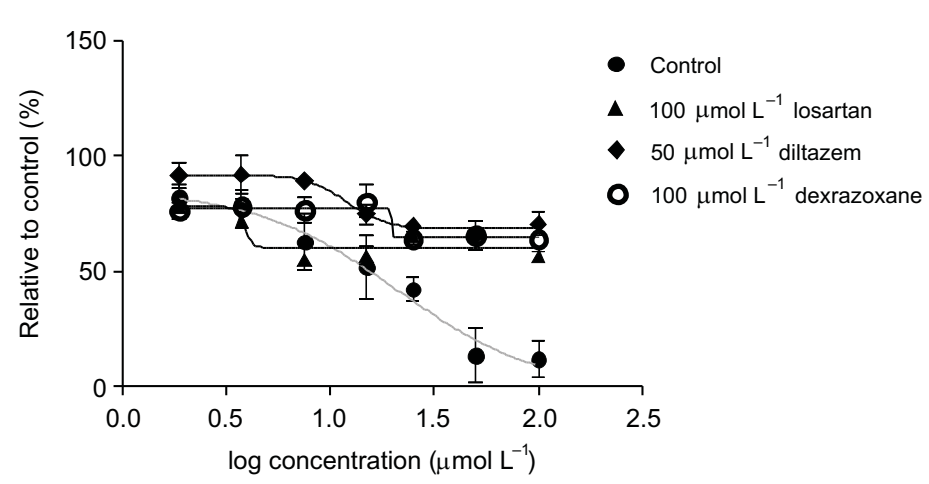

Fig. 5. Effect of anti-ischemia drugs, losartan and diltiazem, in the presence of doxorubicin after $72 \mathrm{~h}$ of incubation.

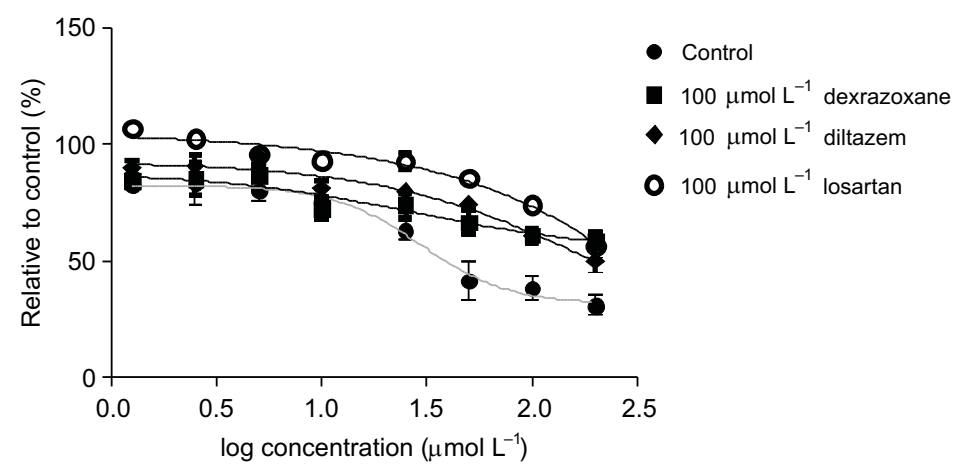

Fig. 6. Effect of anti-ischemia agents on cell survival when exposed to irinotecan for 72 hours.

cytoprotection studies. This was achieved by investigating the effect of 10, 50 and 100 $\mu \mathrm{mol} \mathrm{L} \mathrm{L}^{-1}$ of dexrazoxane, losartan and diltiazem on doxorubicin and irinotecan-induced cytotoxicity (Figs. 3 and 4). The observed effect was not evident at the $10 \mu \mathrm{mol} \mathrm{L}^{-1}$ concentration. Therefore in subsequent cytoprotection studies only doxorubicin and irinotecan were used using dexrazoxane as control. It was evident that co-incubation of 50 $\mu \mathrm{mol} \mathrm{L}-1$ diltiazem, $100 \mu \mathrm{mol} \mathrm{L}^{-1}$ dexrazoxane, and $100 \mu \mathrm{mol} \mathrm{L}^{-1}$ losartan was able to diminish the effects of doxorubicin on $\mathrm{H} 9 \mathrm{c} 2$ cells in lower concentrations, while still maintaining higher than $50 \%$ viability at higher concentrations (Fig. 5). In fact, when doxorubicin was incubated for $72 \mathrm{~h}$ with $50 \mathrm{~mol} \mathrm{~L}^{-1}$ of diltiazem, there was a $58.7 \pm$ $10.2 \%$ reduction in cell death. Similarly, co-incubation of doxorubicin for $72 \mathrm{~h}$ with 100

mol L $\mathrm{L}^{-1}$ dexrazoxane resulted in $52.2 \pm 11.7 \%$ reduction of cell death. Although less effective in comparison with diltiazem and dexrazoxane, co-incubation with $100 \mu \mathrm{mol} \mathrm{L}^{-1}$ of losartan also resulted in $44.7 \pm 5.4 \%$ reduction in cell death. On the other hand, the anti-ischemia and cytoprotective effects of dexrazoxane, losartan, and diltiazem were less effective against cytotoxicity from irinotecan compared to the effect observed in re- 
T. Feridooni et al:: Cytoprotective potential of anti-ischemic drugs against chemotherapy-induced cardiotoxicity in H9c2 myoblast cell line, Acta Pharm. 63 (2013) 493-503.

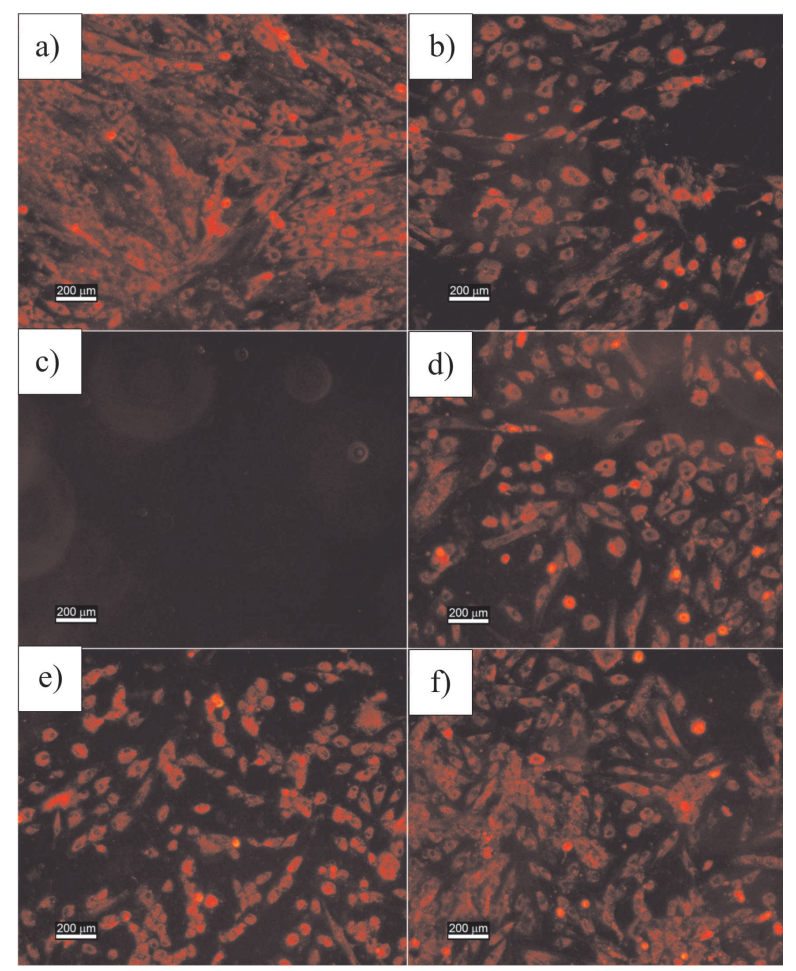

Fig. 7. Fluorescence visualization of cytoprotective effect of dexrazoxane, diltiazem and losartan against doxorubicin-induced toxicity in $\mathrm{H} 9 \mathrm{c} 2$ myoblast cell line and potential cytotoxicity: a) control (PBS), b) irinotecan and c) doxorubicin. Cells incubated with doxorubicin in the presence of: d) dexrazoxane, e) diltiazem and f) losartan.

sponse to doxorubicin (Fig. 6). Co-incubation of irinotecan with $100 \mu \mathrm{mol} \mathrm{L}{ }^{-1}$ of dexrazoxane, losartan or diltiazem reduced cell death by $27.7 \pm 6.9,25.0 \pm 5.1,19.1 \pm 2.3 \%$, respectively.

Fluorescence microscopy allowed visualization of the cytotoxic effect of doxorubicin, compared to the control H9c2 cells (Fig. 7a). There was a significant reduction in H9c2 cell viability after incubation with $100 \mu \mathrm{mol} \mathrm{L}^{-1}$ doxorubicin for $72 \mathrm{~h}$ (Fig. $7 \mathrm{~b}$ ). Although not as potent as doxorubicin (Fig. 7c), incubation of $\mathrm{H} 9 \mathrm{c} 2$ cells with $100 \mu \mathrm{mol} \mathrm{L}^{-1}$ of irinotecan for $72 \mathrm{~h}$ resulted in significant reduction in viable cells. However, co-incubation of the cells with $100 \mu \mathrm{mol} \mathrm{L}-1$ of irinotecan along with $100 \mu \mathrm{mol} \mathrm{L}^{-1}$ of dexrazoxane (Fig. 7d), diltiazem (Fig. 7e), and losartan (Fig. 7f) clearly demonstrated the cytoprotective properties of these drugs. Surprisingly, diltiazem and losartan proved to be more cytoprotective than our control (dexrazoxane) in H9c2 cells against doxorubicin-induced toxicity.

Just like with most successful drugs, discovery of the cytoprotective effect of dexrazoxane was completely serendipitous. The cytoprotective effects of dexrazoxane, in the presence of doxorubicin, have been well established in animal species (11) and hu- 
T. Feridooni et al.: Cytoprotective potential of anti-ischemic drugs against chemotherapy-induced cardiotoxicity in H9c2 myoblast cell line, Acta Pharm. 63 (2013) 493-503.

mans (12). Furthermore, clinical trials have revealed that the cytoprotection of dexrazoxane does not interfere with the anti-tumor effects of doxorubicin, and therefore to this day dexrazoxane serves as one of the best cytoprotective drugs against doxorubicin (13). The hydroxyl radical that makes up the ROS is a highly reactive species that can cause lipid peroxidation, oxidative damage to DNA, and eventual cell death (14). The cytoprotective capability of dexrazoxane is due to its high affinity for LMW-Fe ${ }^{+2}$, which are produced due to the formation of doxorubicin-iron complexes and the subsequent production of ROS and hydroxyl radicals (15). The iron-chelating capability of dexrazoxane was quite evident in the doxorubicin treated H9c2 cells, since it was capable of diminishing the effects of doxorubicin at lower and higher concentrations, as seen in Fig. 5. Although cell death was reduced in the irinotecan treated H9c2 cells, the cytoprotective effects of dexrazoxane were not as potent as seen in the doxorubicin treated group, thus suggesting that the toxicity of irinotecan may be due to other cellular mechanisms in addition to that attributed to $\mathrm{LMW}-\mathrm{Fe}^{+2}$ seen during the formation of doxorubicin-iron complexes which lead to subsequent production of ROS and hydroxyl radicals.

Losartan was the first non-peptide selective angiotensin II receptor blocker approved for clinical use. It is metabolized by cytochrome P450 to the more potent EXP3174 metabolite (16). In addition to its antihypertensive property, losartan has also been shown to have anti-fibrotic capabilities of reducing incidence of cardiac and renal fibrosis. Losartan has been suggested to suppress the effects of transforming growth factor- $\beta 1$ (TGF- $\beta 1$ ) (17). TGF- $\beta 1$ is a cytokine that plays a veriety of roles in cellular processes such as proliferation, differentiation, apoptosis, fibrosis and tumorgenesis (18). Previous studies have shown a significant increase in TGF- $\beta 1$ and 2 receptor expression in doxorubicin induced glomerular toxicity (19). Furthermore, other studies, investigating the cytoprotective effects of doxorubicin-induced rat heart failure, suggested that the protective effects of losartan in doxorubicin-induced heart failure could be due to regulation of circulating angiotensin (1-7) and myocardial angiotensin (1) receptor expression (20). We were also able to show similar findings in our doxorubicin and irinotecan treated H9c2 cells. It is evident from Figs. 5 and 6 that $100 \mu \mathrm{mol} \mathrm{L}^{-1}$ of losartan is capable of significantly diminishing doxorubicin and irinotecan induced toxicity, which could be due to its ability to reduce the effects of TGF- $\beta 1$ or its expression.

Diltiazem is a benzothiazepine calcium channel blocker with special selectivity for vascular calcium channels (21). Contradictory to our findings, diltiazem seems to enhance the cytotoxicity of doxorubicin in Ehrlich ascites carcinoma cells (22). A mode of doxorubicin's cytotoxicity is believed to be due to induction of calcium cycling in cardiac mitochondria. In fact, evidence has been provided by Revis and Marusic (23) and Bachman and Zbinden (24) that doxorubicin reduces the net amount of calcium $\left(\mathrm{Ca}^{2+}\right)$ accumulation in the mitochondria. Further, $\mathrm{Ca}^{2+}$ influx increase in mitochondria could lead to loss of mitochondrial membrane potential, causing cytochrome-c release and subsequent caspase activation (25). Interestingly, the mitochondrial shift towards a more oxidized state took place parallel with significant mitochondrial depolarization that occurred concurrently with increased mitochondrial $\mathrm{Ca}^{2+}$ levels (26). It has been well established that the increase in $\mathrm{Ca}^{2+}$ levels and drop in mitochondrial membrane potential are associated with the production of ROS and that both steps occur prior to the induction of mitochondrial permeability transition and subsequent cell death (27). We have 
T. Feridooni et al.: Cytoprotective potential of anti-ischemic drugs against chemotherapy-induced cardiotoxicity in H9c2 myoblast cell line, Acta Pharm. 63 (2013) 493-503.

shown that by blocking the calcium channels of H9c2 cells using diltiazem one can significantly reduce the influx of extra-cellular $\mathrm{Ca}^{2+}$, which minimizes the detrimental effects of doxorubicin.

Diltiazem was also the most potent drug that reduced cell death when co-administered with irinotecan. Although there has been no report of an increase in intracellular $\mathrm{Ca}^{2+}$ levels from irinotecan, our findings suggest that similarly to doxorubicin, irinotecan may also increase intracellular $\mathrm{Ca}^{2+}$ levels. From the three cytoprotective drugs used in co-treatment with irinotecan, diltiazem was the most effective in diminishing irinotecan-induced H9c2 cell death. Perhaps future studies could focus on $\mathrm{Ca}^{2+}$ levels of cells after treatment with irinotecan to determine whether there is a $\mathrm{Ca}^{2+}$ influx or increase associated with irinotecan.

\section{CONCLUSIONS}

We investigated the effects of anti-ischemia drugs, losartan and diltiazem, as well as dexrazoxane, which have been shown to provide long-term cytoprotection without affecting the efficacy of doxorubicin (28). Dose response experiments for cytoprotective effect were performed using rat myoblast $(\mathrm{H} 9 \mathrm{c} 2)$ cells in the presence of doxorubicin and irinotecan, and the listed cytoprotective and anti-ischemia drugs. We chose H9c2 due to its availability and the fact that it is of cardiac origin, and has been previously used to investigate doxorubicin-induced hypertrophy and cardiac apoptosis (29). In summary, our results suggest that the use of cytoprotective or anti-ischemia drugs (dexrazoxane, losartan and diltiazem) with cytotoxic anticancer drugs (doxorubicin and irinotecan) could have an attenuating effect on cardiac toxicity. Use of these protective agents appeared more effective for doxorubicin compared to irinotecan treated H9c2 cells. It is imperative to note that these drugs can only ablate one or two detrimental effects of these cardiotoxic anticancer drugs, and future studies should focus on therapies involving different combinations of drugs that target different detrimental effects. Combined use of cytoprotective or anti-ischemia drugs in cancer therapies, particularly with those known to cause adverse cardiac events, could improve patient compliance, reduce mortality and healthcare costs in the future.

Acknowledgments. - Funding was provided by the Pharmacy Endowment Fund, Dalhousie University.

\section{REFERENCES}

1. W. A. Denny, Emerging DNA topisomerase inhibitors as anticancer drugs, Expert Opin. Emerg. Drugs 9 (2004) 105-133; DOI: 10.1517/eoed.9.1.105.32948.

2. L. F. Liu, DNA topoisomerase poisons as antitumor drugs, Annu. Rev. Biochem. 58 (1989) 351375; DOI: 10.1146/annurev.bi.58.070189.002031.

3. G. Takemura and H. Fujiwara, Doxorubicin-induced cardiomyopathy from the cardiotoxic mechanisms to management, Prog. Cardiovasc. Dis. 49 (2007) 330-352; DOI: 10.1016/j.pcad.2006. 10.002 . 
T. Feridooni et al:: Cytoprotective potential of anti-ischemic drugs against chemotherapy-induced cardiotoxicity in H9c2 myoblast cell line, Acta Pharm. 63 (2013) 493-503.

4. S. Hitchcock-Bryan, R. Gelber, J. R. Cassady and S. E. Sallan, The impact of induction anthracycline on long-term failure-free survival in childhood acute lymphoblastic leukemia, Med. Pediatr. Oncol. 14 (1986) 211-215; DOI: http:/ /dx.doi.org/10.1002/mpo.2950140405.

5. T. Feridooni, A. Hotchkiss, S. Remley-Carr, Y. Saga and K. B. S. Pasumarthi, Cardiomyocyte specific ablation of p53 is not sufficient to block doxorubicin induced cardiac fibrosis and associated cytoskeletal changes, PLoS ONE 6 (2011) e22801; DOI: 10.1371/journal.pone.0022801.

6. X. Z. Han, S. Gao, Y. N. Cheng, Y. Z. Sun, W. Liu, L. L. Tang and D. M. Ren, Protective effect of naringenin-7-O-glucoside against oxidative stress induced by doxorubicin in H9c2 cardiomyocytes, Biosci. Trends 6 (2012) 19-25; DOI: 10.5582/bst.2012.v6.1.19.

7. B. Glimelius, Benefit-risk assessment of irinotecan in advanced colorectal cancer, Drug Saf. 28 (2005) 417-433; DOI: http:/ /dx.doi.org/10.2165/00002018-200528050-00005.

8. J. Weekes, A. K.-Y. Lam, S. Sebesan and Y.-H. Ho, Irinotecan therapy and molecular targets in colorectal cancer: a systemic review, World J. Gastroenterol. 15 (2009) 3597-3602; DOI: http://dx. doi.org/10.3748/wjg.15.3597.

9. G. Minotti, P. Menna, E. Salvatorelli, G. Cairo and L. Gianni, Anthracyclines: molecular advances and pharmacologic developments in antitumor activity and cardiotoxicity, Pharmacol. Rev. 56 (2004) 185-229; DOI: 10.1124/pr.56.2.6.

10. R. Nithipongvanitch, W. Ittarat, M. P. Cole, J. Tangpong, D. K. S. Clair and T. D. Oberley, Mitochondrial and nuclear p53 localization in cardiomyocytes: redox modulation by doxorubicin (Adriamycin)?, Antioxid. Redox Signal. 9 (2007) 1001-1008; DOI: 10.1089/ars.2007.1632.

11. E. H. Herman and V. J. Ferrans, Amelioration of chronic anthracycline cardiotoxicity by ICRF-187 and other compounds, Cancer Treat Rev. 14 (1987) 225-259; DOI: 10.1016/0305-7372(87) 90011-9.

12. S. M. Swain, F. S. Whaley, M. C. Gerber, M. S. Ewer, J. R. Bianchine and R. A. Gams, Delayed administration of dexrazoxane provides cardioprotection for patients with advanced breast cancer treated with doxorubicin-containing therapy, J. Clin. Oncol. 15 (1997) 1333-1340.

13. S. M. Swain and P. Vici, The current and future role of dexrazoxane as a cardioprotectant in anthracycline treatment: expert panel review, J. Cancer Res. Clin. Oncol. 130 (2004) 1-7; DOI: 10. 1007/s00432-003-0498-7.

14. M. S. Wolin, Interactions of oxidants with vascular signaling systems, Arterioscl. Throm. Vas. Biol. 20 (2000) 1430-1442; DOI: 10.1161/01.ATV.20.6.1430.

15. D. B. Sawyer, R. Fukazawa, M. A. Arstall and R. A. Kelly, Daunorubicin-induced apoptosis in rat cardiac myocytes is inhibited by dexrazoxane, Circ. Res. 84 (1999) 257-65; DOI: 10.1161/01. RES.84.3.257.

16. J. Bierau, R. Leen, A. H. van Gennip, H. N. Caron and A. B. P. van Kuilenburg, Determination of the deoxycytidine kinase activity in cell homogenates with a non-radiochemical assay using reversed-phase high performance liquid chromatography; Identification of a novel metabolite of 2-chlorodeoxyadenosine, J. Chromatogr. B 805 (2004) 339-346; DOI: 10.1016/j.jchromb.2004.03. 036.

17. B. Diop-Frimpong, V. P. Chauhan, S. Krane, Y. Boucher and R. K. Jain, Losartan inhibits collagen I synthesis and improves the distribution and efficacy of nanotherapeutics in tumors, Proc. Natl. Acad. Sci. USA 108 (2011) 2909-2914; DOI: 10.1073/pnas.1018892108.

18. C. E. Runyan, H. W. Schnaper and A.-C. Poncelet, The role of internalization in transforming growth factor beta1-induced Smad2 association with Smad anchor for receptor activation (SARA) and Smad2-dependent signaling in human mesangial cells, J. Biol. Chem. 280 (2005) 83008308; DOI: 10.1074 /jbc.M407939200.

19. K. Tamaki, S. Okuda, T. Ando, T. Iwamoto, M. Nakayama and M. Fujishima, TGF-beta 1 in glomerulosclerosis and interstitial fibrosis of adriamycin nephropathy, Kidney Int. 45 (1994) 525536. 
T. Feridooni et al:: Cytoprotective potential of anti-ischemic drugs against chemotherapy-induced cardiotoxicity in H9c2 myoblast cell line, Acta Pharm. 63 (2013) 493-503.

20. W. Zong, X. Yang, X. Chen, H. Huang, H. Zheng, X. Qin, Y. Yong, K. Cao, J. Huang and X. Lu, Regulation of angiotensin-(1-7) and angiotensin II type 1 receptor by telmisartan and losartan in adriamycin-induced rat heart failure, Acta Pharmacol. Sin. 32 (2011) 1345-1350; DOI: 10.1038/ aps.2011.96.

21. L.-Y. Chiu, J.-L. Ko, Y.-J. Lee, T.-Y. Yang, Y.-T. Tee and G.-T. Sheu, L-type calcium channel blockers reverse docetaxel and vincristine-induced multidrug resistance independent of ABCB1 expression in human lung cancer cell lines, Toxicol. Lett. 192 (2010) 408-418; DOI: 10.1016/j. toxlet.2009.11.018.

22. O. A. Al-Shabanah, A. M. Osman, M. M. Al-Harbi, A. M. Al-Bekairi, N. M. Al-Gharably and S. A. Aziz, Diltiazem potentiation of doxorubicin cytotoxicity and cellular uptake in Ehrlich ascites carcinoma cells, Chemotherapy 41 (1995) 368-377; DOI: 10.1159/000239369.

23. N. W. Revis and N. Marusić, Effects of doxorubicin and its aglycone metabolite on calcium sequestration by rabbit heart, liver, and kidney mitochondria, Life Sci. 25 (1979) 1055-1063; DOI: 10.1016/0024-3205(79)90591-5.

24. E. Bachmann and G. Zbinden, Effect of doxorubicin and rubidazone on respiratory function and $\mathrm{Ca}^{2+}$ transport in rat heart mitochondria, Toxicol. Lett. 3 (1979) 29-34; DOI: 10.1016/03784274(79)90103-6.

25. X. Tan, D.-B. Wang, X. Lu, H. Wei, R. Zhu, S.-S. Zhu, H. Jiang and Z.-J. Yang, Doxorubicin induces apoptosis in $\mathrm{H} 9 \mathrm{c} 2$ cardiomyocytes: role of overexpressed eukaryotic translation initiation factor 5A, Biol. Pharm. Bull. 33 (2010) 1666-1672; DOI: 10.1248/bpb.33.1666.

26. A. V. Kuznetsov, R. Margreiter, A. Amberger, V. Saks and M. Grimm, Changes in mitochondrial redox state, membrane potential and calcium precede mitochondrial dysfunction in doxorubicin-induced cell death, Biochim. Biophys. Acta 1813 (2011) 1144-1152; DOI: 10.1016/j.bbamcr. 2011.03.002.

27. F. De Oliveira, C. Chauvin, X. Ronot, M. Mousseau, X. Leverve and E. Fontaine, Effects of permeability transition inhibition and decrease in cytochrome c content on doxorubicin toxicity in K562 cells, Oncogene 25 (2006) 2646-2655; DOI: 10.1038/sj.onc.1209293.

28. S. E. Lipshultz, R. E. Scully, S. R. Lipsitz, S. E. Sallan, L. B. Silverman, T. L. Miller, E. V. Barry, B. L. Asselin, U. Athale, L. A. Clavell, E. Larsen, A. Moghrabi, Y. Samson, B. Michon, M. A. Schorin, H. J. Cohen, D. S. Neuberg, E. J. Orav and S. D. Colan, Assessment of dexrazoxane as a cardioprotectant in doxorubicin-treated children with high-risk acute lymphoblastic leukaemia: long-term follow-up of a prospective, randomised, multicentre trial, Lancet Oncol. 11 (2010) 950-961; DOI: 10.1016/S1470-2045(10)70204-7.

29. K. E. Merten, Y. Jiang, W. Feng and Y. J. Kang, Calcineurin activation is not necessary for Doxorubicin-induced hypertrophy in $\mathrm{H} 9 \mathrm{c} 2$ embryonic rat cardiac cells: involvement of the phosphoinositide 3-kinase-Akt pathway, J. Pharmacol. Exp. Ther. 319 (2006) 934-940; DOI: 10.1124/jpet. 106.108845. 